\title{
El aprendizaje-servicio como pedagogía durante la pandemia. El proyecto "UCA te da la Mano"
}

\author{
María Brenlla, Estefanía Buzzini, Gabriela González, Claudia Gómez, \\ Juan Hermida, Cristina Lamas, Fernanda Pereyra y María García \\ Pontificia Universidad Católica de Argentina, Argentina
}

\section{Resumen}

La Pontificia Universidad Católica Argentina (UCA) se ha propuesto articular y complementar la formación académica brindada a sus alumnos con una opción de vida auténtica, que los mueva a construir un mundo más justo dando respuestas a las demandas sociales de los sectores vulnerables de la sociedad. A partir de la pandemia ocasionada por el COVID-19, la UCA ha trabajado para dar respuesta a algunas de las nuevas problemáticas que hoy nos afectan. En esa línea se fueron desarrollando estrategias que nos permitieron continuar trabajando con la comunidad aún durante el período de aislamiento social, preventivo y obligatorio. En el marco del Programa "UCA + Comunidad en época de pandemia" surge el proyecto "UCA te da la Mano", realizado conjuntamente por el Departamento de Psicología de la Facultad de Psicología y Psicopedagogía y la Dirección de Compromiso Social y Extensión de la Pontificia Universidad Católica Argentina, el cual tiene el doble objetivo de brindar un espacio de reflexión y orientación para el personal de primera línea de respuesta y ser un espacio de aprendizaje para los alumnos de la carrera de psicología. Participan de ella, docentes, graduados y alumnos.

\section{Palabras clave}

Aprendizaje-servicio, compromiso social, formación universitaria, psicología, personal de primera línea, COVID-19.

Fecha de recepción: 29/IX/2020

Fecha de aceptación: 6/XI/2020

\footnotetext{
Brenlla, M., Buzzini, E., González, G., Gómez, C., Hermida, J., Lamas, C., Pereyra, F. y García, M. (2020). El aprendizaje-servicio como pedagogía durante la pandemia. El proyecto UCA te da la Mano. RIDAS, Revista Iberoamericana de Aprendizaje Servicio, 10, 113-125. DOI10.1344/RIDAS2020.10.10
} 


\title{
Service-learning as a pedagogy during the pandemic. The 'UCA gives you a hand' project
}

\begin{abstract}
One of the main objectives of Pontificia Universidad Católica Argentina (UCA) is to articulate and complement the academic training provided to its students with an authentic lifestyle choice, that would encourage them to build a fairer world by responding to the specific social demands coming from vulnerable sectors of society. The university has thus worked to respond to some of the new problems that now affect us as a society due to the COVID-19 pandemic. As a result, strategies have been developed to allow us to continue working with the community even during this period of social, preventive and compulsory isolation. Within the framework of the program 'UCA + Comunidad en época de pandemia' (UCA + Community in times of a pandemic), UCA has developed a project that we will discuss in this paper: 'UCA te da la mano' (UCA gives you a hand). The project, was jointly carried out by the Department of Psychology of the Faculty of Psychology and Psychopedagogy and the Directorate of Social Commitment and Outreach of the UCA, and it had a dual objective: providing a space for reflection and orientation for first-line response personnel and becoming a learning space for psychology students.
\end{abstract}

\section{Keywords}

Service-learning, social commitment, higher education, psychology, front line staff COVID 19. 


\section{Introducción}

En el contexto actual son varios los factores que inciden sobre la institución universitaria y que le plantean el desafío de adaptarse a las nuevas circunstancias. Nuevas transformaciones, plantean grandes desafíos a la sociedad en general y a la universidad en particular. Problemas cada vez más complejos y globales requieren de una mirada multidisciplinaria que le permita acceder a esas complejidades desde la mayor cantidad de puntos de vistas posibles. Esto lleva a pensar y desarrollar, de manera armoniosa e integral, una política de compromiso y responsabilidad en la sociedad de la cual forma parte.

Ante una situación compleja en la que ningún estado puede dar respuesta a todos los problemas de sus ciudadanos, el compromiso y la participación de las personas en el ámbito de las organizaciones de la sociedad civil son necesarios. Procuran impedir mayores situaciones de exclusión y desigualdad y así poder desarrollar un concepto de inclusión que incorpore derechos de las personas y grupos sociales aún hoy desprotegidos. Para lograr un resultado eficiente se debe identificar y diferenciar los distintos grupos que la integran ya que requieren necesidades de ayuda distintas.

Es por ello, que consideramos que la universidad a través de la docencia, la investigación y la extensión, trabajando de manera articulada, debe ser permeable a aquellas demandas de la sociedad que tengan sentido en función de la misión específica de la Universidad, definida en el punto $\mathrm{N}^{0} 1$ del Ex Corde Ecclesiae como "centro incomparable de creatividad y de irradiación del saber para el bien de la humanidad" (Juan Pablo II, 1990, párr.1).

Las instituciones universitarias en general, y católicas en particular, tienen un papel protagónico en los procesos de desarrollo humano, portando el desafío de arraigarse en la sociedad y contribuir a lograr un mundo más equitativo y justo, ofreciendo una formación humanística y cristiana experiencial a sus alumnos.

El aprendizaje basado en valores y desafíos es un enfoque pedagógico que integra los principios cristianos y humanistas con los desafíos y problemáticas sociales, económicas, políticas y ambientales del entorno en donde se desenvuelven los estudiantes de la UCA. De manera práctica se basa en la integración de problemáticas reales y actuales en donde se plantean diversas alternativas de solución fundamentadas en los valores y en el cuidado del ser humano. Si bien las asignaturas siguen siendo la estructura básica de las carreras profesionales, de manera colegiada se plantean desafíos locales, nacionales y mundiales que, de manera transversal, impactan en los qué y en los cómo aprenden los estudiantes y enseñan los docentes. Se considera que el centro del proceso es la relación estudiante-profesor entorno y con esto se pretende que los alumnos desarrollen un grupo de competencias transversales y disciplinares mediante el planteamiento de desafíos y 
proyectos que impactan en diversas disciplinas y que se abordan siempre desde un punto de vista ético, humanista y de acuerdo con los valores cristianos.

La Dirección de Compromiso Social y Extensión, como área transversal de la Pontificia Universidad Católica Argentina (UCA), tiene por misión generar, junto a las diversas Facultades, iniciativas que permitan reflexionar y concientizar sobre la importancia de una presencia universitaria en las periferias articulando la formación académica (enseñanza y aprendizaje) con la extensión, la investigación y la gestión (Hermida, 2014).

En ese marco, la enseñanza contribuye a que los estudiantes, desde sus itinerarios específicos de aprendizaje, al interactuar con la realidad, afronten problemas y desafíos del mundo actual, comprendiendo en profundidad las dinámicas sociales y sus causas, generando nuevas ideas y recursos que beneficien a todos (Juan Pablo II, 1990).

Tal como lo expresó el papa Francisco (2018) en la Pontificia Universidad Católica de Chile:

El ritmo acelerado y la implantación casi vertiginosa de algunos procesos y cambios que se imponen en nuestras sociedades nos invitan de manera serena, pero sin demora, a una reflexión que no sea ingenua, utópica y menos aún voluntarista. Lo cual no significa frenar el desarrollo del conocimiento, sino hacer de la
Universidad un espacio privilegiado para practicar la gramática del diálogo que forma encuentro (párr.4).

Este camino se viene transitando desde hace ya unos años en la universidad a través de distintas metodologías con el propósito de que los universitarios se formen de manera integral. Formar profesionales expertos en humanidad como nos propone el Proyecto Institucional (Pontificia Universidad Católica Argentina, 2018), requiere garantizar el aprender a ser, aprender a conocer, aprender a hacer, y aprender a vivir juntos (Organización de las Naciones Unidas para la Educación, la Ciencia y la Cultura [UNESCO], 1994).

Al surgir el aislamiento social, preventivo y obligatorio frente a la pandemia generada por el COVID-19, se vio la necesidad de reinventarse con el objetivo de diseñar junto a la comunidad propuestas que den respuesta a los desafíos actuales que nos toca enfrentar como sociedad y continuar brindando espacios de formación en contacto con la realidad a los universitarios.

Con motivo de la convocatoria de la Dirección de Compromiso Social y Extensión al claustro docente de las distintas facultades en el mes de abril surgió la propuesta de desarrollar y llevar adelante el proyecto "UCA te da la mano".

El objetivo de este artículo es dar a conocer dicho proyecto, que consiste en brindar al personal de primera línea en la respuesta un espacio de reflexión y de orientación frente a la vivencia de la 
situación de la pandemia generada por el COVID-19. Se elaboró este proyecto teniendo en consideración que el personal de primera línea en la respuesta conforma un grupo de personas que se encuentra particularmente expuesto a situaciones de gran exigencia, que pueden provocar tensión y preocupación.

\section{Marco teórico}

En el contexto de pandemia las personas suelen sentir estrés y preocupación siendo que el miedo ocupa un lugar central entre las manifestaciones emocionales. Aunque el miedo es un factor de preservación, cuando el sujeto se siente impotente frente a lo que le genera temor, esta emoción puede ocasionar respuestas defensivas poco eficaces. Otros efectos emocionales generados por la situación de pandemia se vinculan a la discriminación, la deshumanización y la disminución de la empatía (Bavel et al., 2020).

Las respuestas más comunes tanto en sujetos directa o indirectamente expuestos por las tareas laborales son el miedo a enfermar o morir; a no poder trabajar durante el aislamiento; a ser despedidos de su trabajo; a ser excluidos socialmente por ser asociados con la enfermedad; a sentirse impotentes para proteger a sus seres queridos o a perderlos y a padecer sentimientos de impotencia, de soledad y de depresión debido al aislamiento (Petzold, Plag y Ströhle, 2020). La literatura disponible describe factores de estrés específicos del brote de COVID-19 que incluyen el riesgo a estar infectado e infectar a otros; la confusión de síntomas comunes con otras enfermedades; la preocupación por el bienestar de los familiares debido a los cambios ocasionados por el aislamiento; y preocupaciones por no poder acceder a la asistencia médica adecuada (Inter-Agency Standing Commitee [IASC], 2020). Los trabajadores de la salud pueden experimentar otros factores de estrés adicionales como la estigmatización debido a su trabajo con pacientes infectados, la posibilidad de contagiar a sus familiares; el hecho de estar sometidos a medidas estrictas de bioseguridad, la mayor demanda laboral y la reducción de la posibilidad de recibir apoyo social debido a los intensos horarios laborales (Petzold et al., 2020).

Otras publicaciones discriminan entre los estresores durante el horario de trabajo y el retorno a los hogares. Incluyen la desorganización frente al caos; sentimientos de inadecuación y de impotencia; omnipotencia; frustración e identificación con las víctimas y con sus familiares durante los horarios de trabajo. Al volver a casa se registró en los trabajadores de primera línea signos de tristeza, miedo, culpa, ansiedad, reacciones somáticas y dificultades para tranquilizarse y relajarse (EMDR Europe Association, 2020).

La literatura relacionada a los aspectos psicosociales asociados a la pandemia por COVID-19, aunque incipiente y escasa, deja entrever el valor de la cooperación, del aprendizaje social y de la percepción de la identidad como formas protectoras, sobre todo para las 
personas que trabajan en la primera línea de respuesta (Bavel et al., 2020).

Como respuesta de la Universidad Católica Argentina a la comunidad, nos propusimos generar un espacio de orientación para el impacto emocional que pueda ocasionar la pandemia por la infección por COVID-19 en los trabajadores de primera línea, a saber médicos, enfermeros, choferes de ambulancias, kinesiólogos, administrativos de servicios de urgencias y unidad de cuidados intensivos (UTI), personal de vigilancia, psicólogos, bioquímicos, camilleros y cualquier otro trabajador cuya función sea de primera necesidad.

Al mismo tiempo, la realización del presente proyecto permitió continuar brindando oportunidades a los universitarios de formarse, desde sus itinerarios específicos de aprendizaje, en contacto con la realidad, afrontando los problemas y desafíos del mundo actual, bajo la pedagogía del aprendizaje-servicio. Esta pedagogía permite a los alumnos ofrecer un servicio a la comunidad, y por otro, aprender de la acción; conectando lo que se aprende de esa acción con el conocimiento ya establecido. Asimismo, esta experiencia de aprendizaje forma la base para la observación y la reflexión en donde el estudiante tiene la oportunidad de observar y desarrollar criterio para actuar y de proponer alternativas de mejora.

Este tipo de aprendizaje se enlaza de manera natural con los valores de la universidad. La propuesta de formación integral humanística y cristiana que propone la UCA a sus estudiantes es brindarles las herramientas como futuros profesionales y ciudadanos comprometidos con los valores que promueve una universidad católica a través de la formación humana, personal y social.

En este sentido, la propuesta pedagógica del aprendizaje-servicio contribuye a superar una visión individualista de la profesión, y a formar graduados comprometidos de manera solidaria y evangélica con las necesidades de su país y de su comunidad.

\section{Experiencia}

El proyecto tiene como objetivo ofrecer al personal de primera línea en la respuesta, un espacio de reflexión y de orientación frente a la vivencia de la situación de la pandemia por COVID19. Para ello, el Programa ofrece dos modalidades de colaboración:

- Un espacio de talleres de orientación online para aliviar el impacto emocional;

- Envío de material gráfico psicoeducativo con información protectora de la Salud Mental.

El primer contacto de las personas con el Programa se realiza mediante la Evaluación Inicial que se realiza de acuerdo con el diseño elaborado en el marco de la cátedra Técnicas e Evaluación psicológicas I de la carrera de psicología. Se aplica la Escala de Malestar Psicológico de Kessler (K-10), ítems construidos ad-hoc para evaluar Miedos y la Escala de Ansiedad Estado de Spielberger. Todas las escalas se 
aplican mediante un formulario digital. El objetivo de esta evaluación es detectar de manera rápida, fiable y válida el malestar emocional vinculado a estados de miedo, de ansiedad y/o de tristeza y depresión en el personal que trabaja en la primera línea de respuesta.

Luego de esta primera etapa, el sujeto puede optar por participar en los Talleres de Orientación o también se le presenta la opción de recibir información psicoeducativa por medios electrónicos.

La segunda etapa se desarrolla a través de talleres de orientación coordinados por docentes de la carrera de psicología con amplia experiencia clínica y capacitación específica. Son acompañados por graduados de la carrera.

La intervención fue diseñada por directivos y docentes de la facultad y la modalidad de trabajo fue plasmada en un Manual de Procedimientos. El mismo incluye material de trabajo para los talleres, el orden secuencial de las diferentes actividades, y las consignas para llevarlas a cabo.

El Programa también incluye la participación de alumnos del último año de la carrera de Psicología, como observadores no participantes en el marco de sus prácticas profesionales. De esta manera, el dispositivo se propone aportar orientación frente al impacto emocional para los agentes de primera línea de respuesta, y al mismo tiempo, a la formación de los estudiantes en la adquisición de destrezas profesionales.
Se confeccionó un esquema organizativo dividido en tres instancias: el entrenamiento de coordinadores de los talleres, el ingreso y asignación de talleres a las personas interesadas y la etapa propiamente de los talleres.

Figura 1. Esquema organizativo

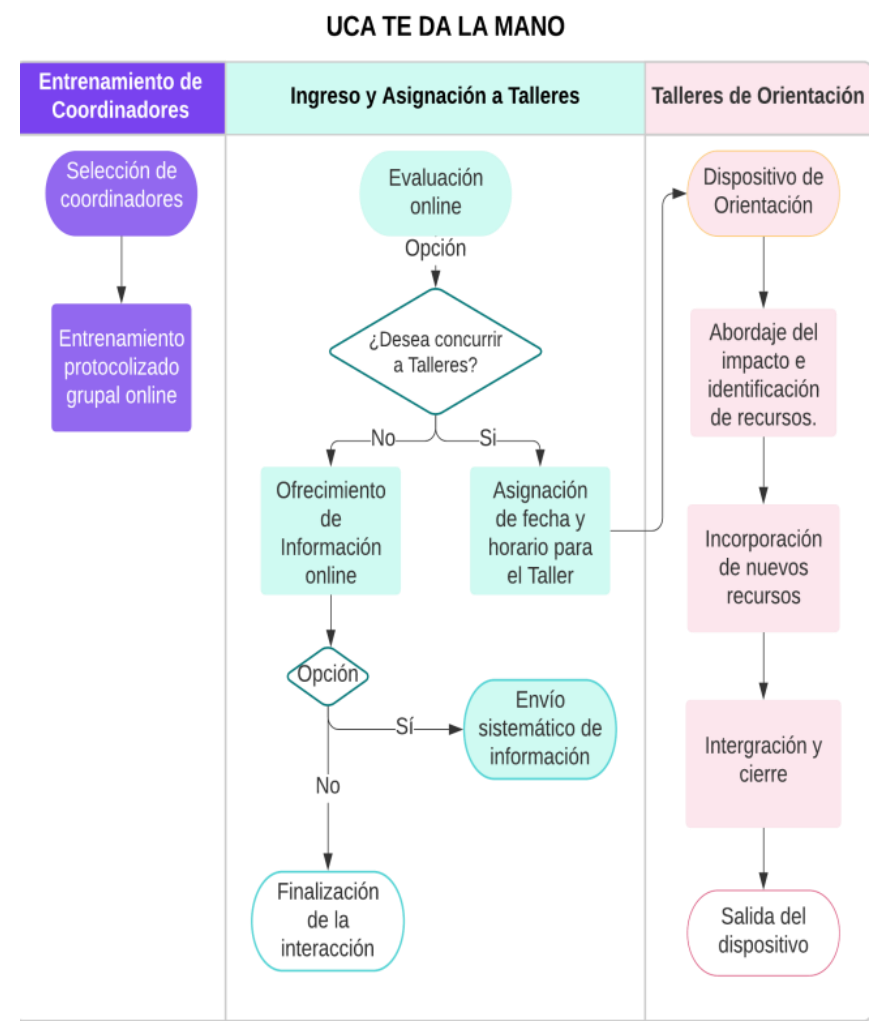

Fuente: elaboración propia

\section{Talleres de orientación}

Los talleres con modalidad online, a través de la plataforma zoom, tienen por objetivo brindar un espacio de orientación para compartir experiencias, abordar el impacto emocional e identificar recursos de afrontamiento. 
Consiste en tres encuentros de una duración de 1 hora 30 minutos cada uno, con una frecuencia quincenal. Se ofrecen en una variedad de días y horarios, para garantizar que todos aquellos que estén interesados pueden encontrar una banda horaria que se adapte a sus necesidades. El equipo está integrado por 2 coordinadores profesionales de la salud mental (un docente y un graduado), un alumno observador y 8 participantes como máximo.

Los encuentros se centran en la promoción de comportamientos saludables e incluyen estrategias dirigidas a la reducción del miedo y del estigma; a la modificación de estrategias de afrontamiento ineficaces y al reconocimiento de las propias fortalezas, a la facilitación del diálogo y de la contención grupal, a la reducción de la activación fisiológica; a la reestructuración cognitiva y la regulación emocional. Previo al inicio de estos se realizaron simulacros con el objetivo de ajustar la propuesta en cuanto a duración, cantidad de participantes y contenidos.

La actividad del primer día de taller se centra en compartir las experiencias relacionadas con su labor asociado a la respuesta frente a la pandemia, el reconocimiento del impacto emocional y la identificación de las estrategias de afrontamiento. Se busca la facilitación del diálogo y de la actitud de compartir con otros como medio de promover la contención grupal y la estimulación de modos de afrontamiento más eficaces y saludables.
La segunda reunión continúa el trabajo, pero de un modo más directivo, utilizando herramientas cognitivas de relajación, de resolución de problemas y de toma de decisiones para la estructuración de actividades de taller que permitan el aprendizaje comunitario de estrategias adaptativas y flexibles para el afrontamiento de situaciones problemáticas a las que las personas se enfrentan cotidianamente.

La tercera reunión tiene como objetivo la integración y la asimilación de lo trabajado en los dos días de taller a las modalidades personales de cada integrante. Esta reunión propicia un cierre y la reflexión sobre lo que fue trabajado en los talleres, la comparación de los propios recursos antes y después de la participación en el programa y la facilitación del uso de los recursos adquiridos y reconocidos como propios durante el trabajo de taller.

Como se mencionó anteriormente, aquellos que no desean participar de los talleres, pueden optar por recibir material psicoeducativo. El mismo fue seleccionado por personal de la salud mental usando como fuente los datos oficiales sobre la pandemia por COVID19 y los ofrecidos por los organismos internacionales. Tiene el propósito de brindar información acerca de comportamientos preventivos que estimulen el bienestar psicosocial durante la situación de pandemia.

La siguiente tabla refleja una síntesis de los objetivos y propuestas de los diferentes encuentros. 
Tabla 1. Objetivos y propuestas por taller

\begin{tabular}{|c|c|c|}
\hline \multicolumn{3}{|c|}{ Taller 1} \\
\hline Objetivo & Propuesta & Recursos \\
\hline $\begin{array}{l}\text { I. Compartir } \\
\text { experiencias } \\
\text { respecto a sus } \\
\text { tareas en } \\
\text { relación a la } \\
\text { pandemia. } \\
\text { II. Reconocer las } \\
\text { diferentes } \\
\text { respuestas } \\
\text { emocionales y de } \\
\text { comportamiento. }\end{array}$ & \multirow{2}{*}{$\begin{array}{l}\text { Presentación de } \\
\text { consignas e } \\
\text { imágenes que } \\
\text { facilitan la } \\
\text { participación } \\
\text { grupal. }\end{array}$} & \multirow{2}{*}{$\begin{array}{c}\text { Plataforma } \\
\text { Zoom }\end{array}$} \\
\hline $\begin{array}{l}\text { III. Transmitir } \\
\text { información } \\
\text { sobre la } \\
\text { normalidad de } \\
\text { las reacciones } \\
\text { emocionales a } \\
\text { situaciones de } \\
\text { stress. } \\
\text { IV. Identificar } \\
\text { recursos de } \\
\text { afrontamiento. }\end{array}$ & & \\
\hline \multicolumn{3}{|c|}{ Taller 2} \\
\hline Objetivo & Propuesta & Recursos \\
\hline $\begin{array}{l}\text { I. Compartir } \\
\text { experiencias, } \\
\text { respuestas } \\
\text { emocionales } \\
\text { concomitantes y } \\
\text { modos de } \\
\text { afrontamiento. } \\
\text { II. Reconocer las } \\
\text { manifestaciones } \\
\text { corporales y } \\
\text { cognitivas de } \\
\text { ansiedad y } \\
\text { stress. } \\
\text { III. Aportar } \\
\text { recursos y } \\
\text { estrategias para } \\
\text { el manejo de la } \\
\text { ansiedad y el } \\
\text { stress. }\end{array}$ & $\begin{array}{c}\text { Puesta en } \\
\text { práctica de } \\
\text { ejercicios de } \\
\text { control de stress. } \\
\\
\text { A partir de } \\
\text { imágenes se } \\
\text { identifican } \\
\text { recursos propios } \\
\text { de afrontamiento } \\
\text { y autocuidado. }\end{array}$ & $\begin{array}{c}\text { Plataforma } \\
\text { Zoom }\end{array}$ \\
\hline
\end{tabular}

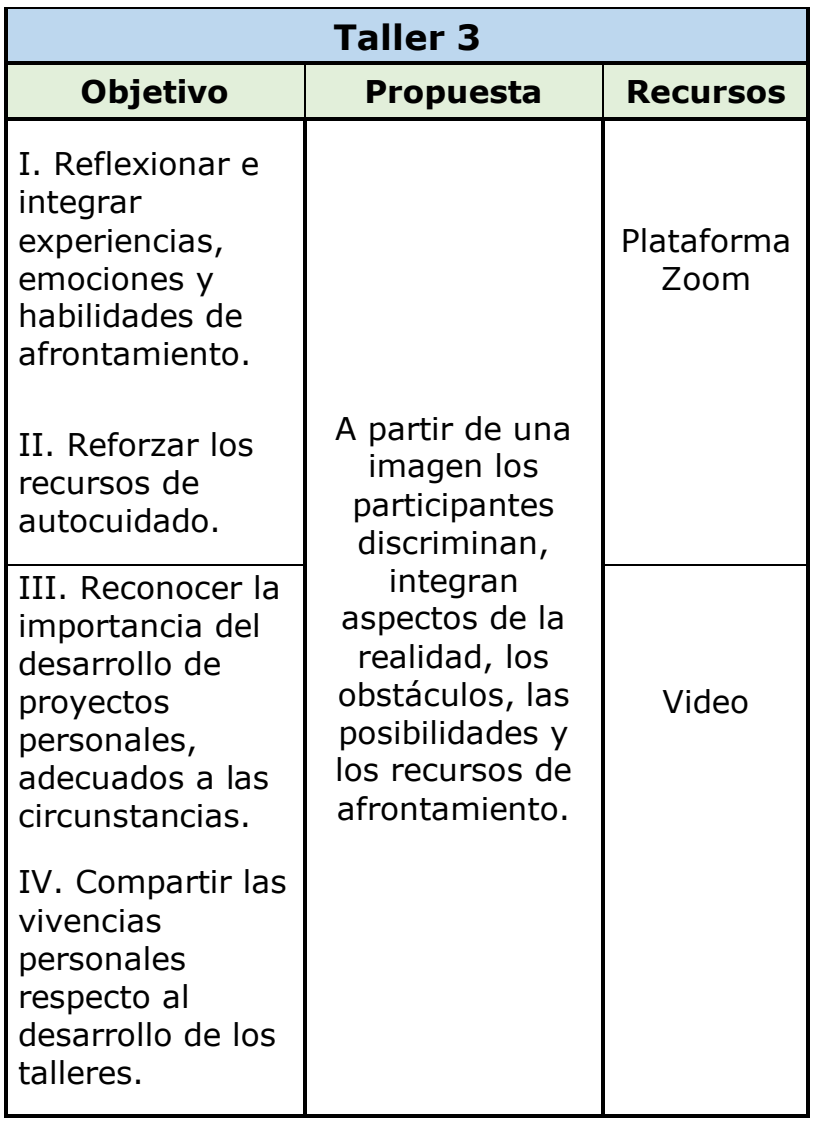

Fuente: elaboración propia

Con el objetivo de orientar a los alumnos en la integración de contenidos temáticos de la currícula de la carrera, y el aprendizaje de habilidades, se propone la utilización de una guía de observación de los talleres. En la misma deben volcar observaciones acerca de las manifestaciones emocionales que presentan los participantes, la dinámica de cada taller, las intervenciones de los coordinadores y la autobservación de su propia experiencia como observador no participante.

Asimismo, luego de cada encuentro se destina un espacio para el intercambio entre los alumnos y los miembros del 
equipo coordinador.

\section{Resultados preliminares de la evaluación inicial}

\subsection{Caracterización de los participantes}

Con el objetivo de brindar una descripción general de los resultados hasta el momento se indica que respondieron a los cuestionarios 28 personas que se desempeñan en tareas en primera línea de atención por COVID-19 (19 mujeres y 9 varones).

Las edades oscilan entre 20 y 70 años $(\mathrm{M}=46,0 ; \mathrm{DE}=14,6)$ y el $80 \%$ indica vivir con su pareja y/o familiares. Catorce $(50 \%)$ de los entrevistados señalan mantener contacto directo con pacientes con COVID-19 mientras que el resto, en su mayoría, se dedica al cuidado de personas en riesgo (por ejemplo, atención de personas mayores) y solo 4 a tareas sin relación con el COVID-19. Las personas que solicitaron la participación en el taller pertenecen a los dos primeros grupos.

\subsection{Capacitación y protección}

En cuanto a las preguntas acerca de la capacitación referida a los protocolos COVID-19, 12 (43\%) indicaron haber recibido suficiente capacitación mientras que 12 (43\%) señaló haber recibido formación, pero de forma insuficiente y $4(16 \%)$ no tenerla. A la vez, el $60 \%$ reportó contar con suficiente equipo de protección personal.

\subsection{Temores de contagio}

La siguiente tabla resume los datos de autopercepción de contagiarse o contagiar a otros de COVID-19 y de sueño y descanso. Como puede notarse, la preocupación más importante estriba en el temor a contagiar en el hogar, en menor medida, en el trabajo, y la percepción de no descansar apropiadamente (cerca de un $47 \%$ ).

Tabla 2. Autopercepciones de temor al contagio de COVID-19 y de descanso y sueño

\begin{tabular}{|ll|c|c|}
\hline $\begin{array}{c}\text { Autopercepciones de } \\
\text { temor al contagio y de } \\
\text { descanso y sueño }\end{array}$ & $\mathbf{f}$ & \% \\
$\mathbf{N = 2 8}$ & Nada & $\mathbf{9}$ & $\mathbf{3 2 , 1}$ \\
\hline Contagiar_trabajo & Poco & $\mathbf{1 0}$ & $\mathbf{3 5 , 7}$ \\
& Bastante & $\mathbf{9}$ & $\mathbf{3 2 , 1}$ \\
& Mucho & $\mathbf{0}$ & $\mathbf{0}$ \\
\hline & Nada & $\mathbf{6}$ & $\mathbf{2 1 , 4}$ \\
Contagiar_casa & Poco & $\mathbf{8}$ & $\mathbf{2 8 , 6}$ \\
& Bastante & $\mathbf{1 0}$ & $\mathbf{3 5 , 7}$ \\
& Mucho & $\mathbf{4}$ & $\mathbf{1 4 , 3}$ \\
\hline \multirow{5}{*}{ No_descanso } & Nada & $\mathbf{8}$ & $\mathbf{2 8 , 6}$ \\
& Poco & $\mathbf{7}$ & $\mathbf{2 5}$ \\
& Bastante & $\mathbf{1 0}$ & $\mathbf{3 5 , 7}$ \\
& Mucho & $\mathbf{3}$ & $\mathbf{1 0 , 7}$ \\
\hline
\end{tabular}

Fuente: elaboración propia

\subsection{Percepción de agotamiento laboral}

En relación con este punto, en la tabla 3 se advierten que hay dos tópicos que se perciben con mayor frecuencia: los relacionados al desgaste emocional personal (ítems 1 y 5) y los referidos al agotamiento laboral (ítems 2 y 3). En cambio, los vinculados al contacto directo con la gente o a la frustración fueron los menos indicados. 
Tabla 3. Autopercepciones de desgaste laboral

\begin{tabular}{|c|c|c|c|}
\hline \multicolumn{2}{|c|}{$\begin{array}{l}\text { Autopercepciones de desgaste } \\
\text { laboral - } \mathbf{N}=\mathbf{2 8}\end{array}$} & \multirow{2}{*}{$\begin{array}{c}f \\
15\end{array}$} & \multirow{2}{*}{$\begin{array}{c}\% \\
53,6\end{array}$} \\
\hline \multirow{3}{*}{$\begin{array}{l}\text { 1. Me siento } \\
\text { emocionalmente } \\
\text { sobrepasado en } \\
\text { mi trabajo. }\end{array}$} & $\begin{array}{l}\text { una vez al mes o } \\
\text { menos }\end{array}$ & & \\
\hline & pocas veces al mes & 3 & 10,7 \\
\hline & & 10 & 35,7 \\
\hline \multirow{3}{*}{$\begin{array}{l}\text { 2. Cuando } \\
\text { termino mi } \\
\text { jornada de } \\
\text { trabajo me } \\
\text { siento agotado. }\end{array}$} & & 12 & 42,9 \\
\hline & pocas veces al mes & 4 & 14,3 \\
\hline & $\begin{array}{l}\text { una vez a } \\
\text { más }\end{array}$ & 12 & 42,9 \\
\hline \multirow{3}{*}{$\begin{array}{l}\text { 3. Cuando me } \\
\text { levanto por la } \\
\text { mañana y me } \\
\text { enfrento a otra } \\
\text { jornada de } \\
\text { trabajo me } \\
\text { siento agotado. }\end{array}$} & mer & 16 & 57,1 \\
\hline & pocas veces al & 2 & 7,1 \\
\hline & $\begin{array}{l}\text { una vez a la semana o } \\
\text { más }\end{array}$ & 10 & 35,7 \\
\hline \multirow{3}{*}{$\begin{array}{l}\text { 4. Siento que } \\
\text { trabajar todo el } \\
\text { día con la gente } \\
\text { me cansa. }\end{array}$} & & 17 & 60,7 \\
\hline & pocas veces al mes & 4 & 14,3 \\
\hline & & 7 & 25 \\
\hline \multirow{3}{*}{$\begin{array}{l}\text { 5. Siento que mi } \\
\text { trabajo me está } \\
\text { desgastando. }\end{array}$} & & 13 & 46, \\
\hline & pocas veces al mes & 5 & 17,9 \\
\hline & & 10 & 35,7 \\
\hline \multirow{3}{*}{$\begin{array}{l}\text { 6. Me siento } \\
\text { frustrado por el } \\
\text { trabajo. }\end{array}$} & $\begin{array}{l}\text { una } \\
\text { men }\end{array}$ & 20 & 71,4 \\
\hline & pocas veces al mes & 2 & 7,1 \\
\hline & $\begin{array}{l}\text { una vez a la semana o } \\
\text { más }\end{array}$ & 6 & 21,4 \\
\hline \multirow{3}{*}{$\begin{array}{l}\text { 7. Siento que } \\
\text { estoy demasiado } \\
\text { tiempo en } \mathbf{~ m i} \\
\text { trabajo. }\end{array}$} & $\begin{array}{l}\text { una } \\
\text { men }\end{array}$ & 18 & 64,3 \\
\hline & pocas veces al mes & 2 & 7,1 \\
\hline & $\begin{array}{l}\text { una vez a la semana o } \\
\text { más }\end{array}$ & 8 & 28, \\
\hline \multirow{3}{*}{$\begin{array}{l}\text { 8. Siento que } \\
\text { trabajar en } \\
\text { contacto directo } \\
\text { con la gente me } \\
\text { cansa. }\end{array}$} & $\begin{array}{l}\text { una vez al mes o } \\
\text { menos }\end{array}$ & 20 & 71,4 \\
\hline & pocas veces al mes & 4 & \\
\hline & $\begin{array}{l}\text { una vez a la semana o } \\
\text { más }\end{array}$ & 4 & 14,3 \\
\hline \multirow{3}{*}{$\begin{array}{l}\text { 9.Me siento } \\
\text { como si } \\
\text { estuviera al } \\
\text { límite de mis } \\
\text { posibilidades. }\end{array}$} & $\begin{array}{l}\text { una vez al mes o } \\
\text { menos }\end{array}$ & 19 & 67,9 \\
\hline & pocas veces al mes & 4 & 14,3 \\
\hline & $\begin{array}{l}\text { una vez a la semana o } \\
\text { más }\end{array}$ & 5 & 17,9 \\
\hline
\end{tabular}

Fuente: elaboración propia

\subsection{Percepción de malestar psicológico}

Los resultados obtenidos en la Escala de Malestar Psicológico ( $\mathrm{K}-10)(\mathrm{M}=23$; $\mathrm{DE}=9$ ) son compatibles con la percepción de bajo riesgo de malestar. Vale decir, si bien se reconoce la situación de tensión y estrés que implica la situación COVID-19, los entrevistados cuentan con recursos adaptativos que les permiten sobrellevarla y, en consecuencia, reportan escasamente la aparición de inquietud o desazón.

\subsection{Percepción de estado de ansiedad}

En cuanto a la evaluación referida al estado de ansiedad los resultados muestran valores altos $(M=44,6 ; \mathrm{DE}=$ $12,7)$. Ello es razonable, teniendo en cuenta que se evalúa la presencia de inquietud, nerviosismo y agitación que se perciben "en este momento" $y$, en su mayoría, las personas entrevistadas se encuentran realizando tareas que, de por sí, implican un estado de alerta y atención que puede aumentar los niveles de ansiedad en forma circunscripta en el tiempo.

\section{Conclusiones}

Este artículo pone de manifiesto la presencia de dispositivo como el implementados desde la Universidad constituye un primer contacto por parte de los miembros de la comunidad universitaria con una nueva realidad que ha irrumpido de forma inesperada. Es al mismo tiempo, una oportunidad para descubrir nuevas formas de fraternidad y solidaridad "para que germine un ser humano diferente" que requiere nuestra sociedad.

Brenlla, M., Buzzini, E., González, G., Gómez, C., Hermida, J., Lamas, C., Pereyra, F. y García, M. (2020). 
La participación de los alumnos universitarios en implementación de estos dispositivos les provee de herramientas que no pueden ser asumidas desde el espacio del aula. La experiencia de aprendizaje-servicio propone el desafío de desarrollar múltiples habilidades: ampliar la posibilidad de dar respuesta frente a lo desconocido, generar nuevas categorías para evaluar, planificar nuevos modos de acción frente a un desafío continuo y compartir lo vivenciado, promover la búsqueda de marcos de referencia amplios para la comprensión de sí mismos y del espacio social en que habitan. Estas experiencias, les permiten "pasar del plano de los ideales declamados a prácticas donde los principios de la solidaridad y la justicia deben ser puestos en juego a través de compromisos personales concretos" (Tapia, 2000, p.123).

En la medida que los universitarios van tomando un contacto más fluido con la experiencia desarrollan una conciencia crítica de sus propias prácticas, procurándoles herramientas concretas para la actuación profesional.

Actualmente los diversos ciclos de talleres de orientación continúan desarrollándose y al momento se han realizado 5 ciclos conformados por médicos, kinesiólogos, enfermeros y personal administrativo con tareas en instituciones de salud. Los universitarios han valorado la oportunidad de poder participar como observadores de estos y compartirán en un ateneo con alumnos, docentes y autoridades de la universidad los aprendizajes adquiridos a partir de la participación que han tenido a lo largo del proyecto.

Por otra parte, el personal de primera línea ha realizado devoluciones en las cuales se evidencia que la experiencia ha sido muy enriquecedora para ellos y agradecen la posibilidad de contar con un espacio de contención y orientación, como lo señala el siguiente testimonio:

"...yo estoy bien y puse en marcha todas las herramientas que amablemente y con muy buena disposición me brindaron...Muchísimas gracias. A mí me sirvió y mucho" (Participante grupo 2).

Asimismo, los universitarios participantes han manifestado que estos espacios de formación han sido muy valiosos para ellos:

"Para mí fue una experiencia muy rica, en donde pude poner en práctica lo aprendido teóricamente...considero que, a pesar de ser una práctica virtual, aprendí mucho, tanto de las participantes como de la profesora. Como alumna les agradezco que me brinden esta posibilidad de poder formar parte de este tipo de programas, que no sólo nos ayuda a nosotros en nuestro desarrollo académico, sino que también es un servicio que brinda la facultad que considero muy útil para quienes se encuentran en la primera línea de respuesta en la Pandemia" (Sofía, alumna de la carrera de psicología). Revista Iberoamericana de Aprendizaje Servicio, 10, 113-125. DOI10.1344/RIDAS2020.10.10 


\section{Referencias bibliográficas}

Bavel, J.J.V., Baicker, K., Boggio, P.S., Capraro, V., Cichocka, A., Cikara, M.,... Willer, R. (2020). Using social and behavioural science to support COVID19 pandemic response. Nature Human Behavior, (4), 460-471. doi:

10.1038/s41562-020-0884-z

EMDR Europe Association. (2020). Guidelines for first responders-Selfprotection for first responders and Health Professionals. Recuperado de: https://www.emdr.nl/wpcontent/uploads/2020/03/EMDREurope GUIDELINESFORFIRSTRESPON DERS.pdf

Francisco. (17 de enero de 2018). Visita a la Pontificia Universidad Católica de Chile. Discurso del Santo Padre. Recuperado de:

http://www. vatican.va/content/francesc o/es/speeches/2018/january/document s/papa-francesco 20180117 cilesantiago-pontuniversita.html\# ftn1

Hermida, J. (2014). Universitarios en la periferia. Señales del Cono Sur, (7), 42-45. Recuperado de:

http://senalesdelconosur.com.ar/revista /numero7/

Inter-Agency Standing Commitee [IASC]. (2020). Briefing note on addressing mental health and psychosocial aspects of COVID-19 Outbreak. Version 1.1. Nueva York, Estados Unidos de América: Ochoa. Recuperado de: https://www.drugsandalcohol.ie/31767/ 1/MHPSS COVID19 Briefing Note $2 \mathrm{M}$ arch 2020.pdf
Juan Pablo II. (15 de agosto de 1990). Constitución Apostólica Ex Corde Ecclesiae del Sumo Pontífice Juan Pablo II sobre las Universidades Católicas. Vaticano. Recuperado de: http://www.vatican.va/content/johnpaulii/es/apost constitutions/documents/hf jp-ii apc 15081990 ex-cordeecclesiae.html

Organización de las Naciones Unidas para la Educación, la Ciencia y la Cultura [UNESCO]. (1994). Informe a la UNESCO de la Comisión internacional sobre la educación para el siglo XXI, presidida por Jacques Delors. La Educación encierra un tesoro. Madrid, España: Santillana - Ediciones Unesco.

Petzold, M., Plag, J., y Ströhle, A. (2020). Umgang mit psychischer Belastung bei Gesundheitsfachkräften im Rahmen der Covid-19-Pandemie. Der Nervenarzt. doi:10.1007/s00115020-00905-0

Pontificia Universidad Católica Argentina. (2018). Proyecto institucional 2018-2022. Ciudad Autónoma de Buenos Aires, Argentina. Recuperado de:

http://wadmin.uca.edu.ar/public/ckedit or/Universidad/Proyecto\%20Institucion al/UCA-Proyecto-Institucional-20182022.pdf

Tapia, M.N. (2000). La Solidaridad como Pedagogía. Buenos Aires, Argentina: Ciudad Nueva. Revista Iberoamericana de Aprendizaje Servicio, 10, 113-125. DOI10.1344/RIDAS2020.10.10 